\title{
Sex Hormonal Profile in Men and Women with Epilepsy on Enzyme-Inducing Antiepileptic Drugs: A Case-Control Study
}

\author{
Shivalaxmi Manchukonda ${ }^{1,2}$ Shaik Afshan Jabeen ${ }^{1}$ \\ Priscilla Abraham Chandran²
${ }^{1}$ Department of Neurology, Nizam's Institute of Medical Sciences, Hyderabad, Telangana, India
${ }^{2}$ Department of Biochemistry, Nizam's Institute of Medical Sciences, Hyderabad, Telangana, India
${ }^{3}$ Department of Neurology, Sree Chitra Tirunal Institute for Medical \\ Sciences and Technology, Thiruvananthapuram, Kerala, India \\ Int J Epilepsy 2018;5:38-43
}

Noorjahan Mohammed² Ajith Cherian ${ }^{3}$

\begin{abstract}
Address for correspondence Shaik Afshan Jabeen, MBBS, MD, DM Neurology, Department of Neurology, Nizam's Institute of Medical Sciences, Hyderabad, Telangana, India (e-mail: drjabeennims@ gmail.com).
\end{abstract}

\author{
Abstract \\ Keywords \\ - libido \\ - testosterone \\ - sex hormone-binding \\ globulin \\ - sexual dysfunction \\ - androgen index \\ - enzyme induction \\ - antiepileptic drugs
}

Introduction Prevalence of sexual and reproductive dysfunction in men and women with epilepsy (MWE and WWE) ranges from 20 to $60 \%$ in different studies. Epilepsy and antiepileptic drugs both contribute in varying degrees to the reproductive endocrine dysfunction. Aims and Objectives The aim of this study is to assess the alteration in sex hormone levels in MWE and WWE on enzyme-inducing antiepileptic drugs (EIAEDs).

Materials and Methods This is a cross-sectional case-control study of 29 MWE and 26 WWE on EIAEDs. Control group were age- and sex-matched healthy volunteers. Serum sex hormones such as total testosterone (TT), dehydroepiandrosterone sulfate (DHEAS), estradiol $\left(E_{2}\right)$ and progesterone, gonadotropins such as luteinizing hormone (LH), follicular-stimulating hormone (FSH), and sex hormone-binding globulin (SHBG) levels were estimated. Free testosterone (FT) and bioactive testosterone (BAT) were calculated. Gonadal efficiency in men was estimated by TT/LH and in women by $\mathrm{E}_{2} / \mathrm{SHBG}$ and $\mathrm{LH} / \mathrm{FSH}$ ratios.

Results There was a significant decrease in TT/LH, FT, BAT, DHEAS, and progesterone along with a significant elevation of SHBG and $E_{2}$ in MWE on EIAEDs. There was also significant elevation of gonadotropins such as LH, FSH, and prolactin (PRL) in MWE. A significant decrease in progesterone, DHEAS, and $\mathrm{E}_{2} / \mathrm{SHBG}$ along with a marked elevation in SHBG, LH, FSH, and PRL was found in WWE.

Conclusion This study clearly demonstrates that EIAEDs have an adverse effect on reproductive health in both MWE and WWE.

\section{Introduction}

Epilepsy warrants long-term use of antiepileptic drugs (AEDs). One of the major adverse effects of AEDs is hormonal dysfunction, especially seen with enzyme-inducing AEDs (EIAEDs) that alter reproductive hormone levels in both men and women with epilepsy (MWE and WWE). Prevalence of reproductive and sexual dysfunction in MWE and WWE ranges from 20 to $60 \%$, respectively. ${ }^{1-3}$
EIAEDs can cause variations in sex hormone levels, either by altering their metabolism or by disrupting the hypothalamo-pituitary-gonadal (HPG) axis. ${ }^{4}$ Consistent hormonal changes observed with older AEDs in previous studies include reduced free testosterone (FT), bioactive testosterone (BAT), and dehydroepiandrosterone sulfate (DHEAS) levels, and elevated sex hormone-binding globulin (SHBG) levels. The hormonal levels of gonadotropins (follicular-stimulating hormone [FSH], luteinizing hormone [LH]), prolactin (PRL),
DOI https://doi.org/

$10.1055 / \mathrm{s}-0038-1667210$.

ISSN 2213-6320.
Copyright @2018 Indian Epilepsy Society
License terms

()(1) $\Theta \circledast$ 
total testosterone (TT), estradiol $\left(\mathrm{E}_{2}\right)$, and progesterone levels were found to be variable. ${ }^{5-13}$ The genetic variance in the population studied may have an impact on the prevalence of these abnormalities. There were few studies in WWE in this regard from Indian subcontinent but none with respect to MWE. ${ }^{14}$ We intend to study the effect of EIAED on sex hormone levels and its clinical implications to a certain extent.

\section{Materials and Methods}

The study was conducted in the Department of Neurology in collaboration with the Department of Biochemistry, Nizam's Institute of Medical Sciences, Hyderabad, from May 2013 to July 2014. This was a cross-sectional case-control study of 29 MWE and 26 WWE on EIAEDs. Control group were age- and sex-matched healthy volunteers. The study was approved by the institutional ethical committee (IEC). Informed consent was taken from each participant. For women, the day of the menstrual cycle on which the hormonal analysis was done was noted.

We included MWE and WWE of reproductive age who had been on EIAEDs for at least 1 year. Only those who had been on stable doses for the past 6 months (a time period long enough to bring out the possible reproductive endocrine effects of the AEDs) and who were seizure free for at least 1 year (to remove the effect of recurrent seizures) were selected. The exclusion criteria included patients with obesity, systemic diseases such as diabetes, liver and kidney diseases, thyroid disorders, polycystic ovarian disorders (PCODs), and other comorbid conditions such as depression, anxiety disorder, and mental retardation. Patients with history of alcohol consumption; substance abuse or those using hormones, tranquilizers, and oral contraceptives; and pregnant or lactating women were also excluded.

The control population were normal healthy volunteers. All the above-mentioned exclusion criteria were applied in control patients so as to avoid confounders. An International Index of Erectile Function (IIEF-5) questionnaire to assess the sexual dysfunction (Rosen et al, 1999) was administered to MWE. This questionnaire consists of five questions that deal with various aspects of sexuality. ${ }^{67}$ A score of $\leq 21$ is taken as sexual dysfunction. A score of $\geq 22$ is considered as normal (Supplementary Table 1, available in online version only). This questionnaire is available in multiple languages. We administered it in the local language (Telugu, Hindi, and Urdu).

Women whose blood samples were collected on days 1 to 12 of their menstrual cycle were categorized to be in follicular phase and on days 16 to 35 to be in luteal phase. In the control women, blood samples were collected on the corresponding days of menstrual cycle as taken in patients.

Serum levels of sex hormones and SHBG were estimated by electro-chemiluminescence immunoassay on cobas e 411 (Roche Diagnostics, Risch-Rotkreuz, Switzerland).Serum Albumin was estimated by calorimetric assay (BCG method) on cobas c 501 (Roche Diagnostics). FT and BAT were calculated (Sodergard et al, 1982). Free androgen index (FAI) was calculated as (TT $[\mathrm{nmol} / \mathrm{L}] /$ SHBG $[\mathrm{nmol} / \mathrm{L}]) \times 100$. Gonadal efficiency in men was estimated by TT/LH and in women by $\mathrm{E}_{2} / \mathrm{SHBG}$ and $\mathrm{LH} / \mathrm{FSH}$ ratios.

Statistical analyses were performed using the MedCalc statistical software (version 14.8.1, MedCalc Software Bvba; Ostend, West Flanders, Belgium). Normality of the data was tested by D'Agostino and Pearson test. To test differences between the two groups, that is, controls and patients, we used unpaired Student's $t$-test for normally distributed variables and Mann-Whitney $U$ test for non-normally distributed variables. A $p<0.05$ was considered as statistically significant.

\section{Results}

The demographic variables of study population, which consisted of 29 MWE and 26 WWE (14 in follicular phase and

Table 1 Demographic variables of patient groups

\begin{tabular}{|c|c|c|}
\hline Characteristics & MWE $(n=29)$ & WWE $(n=26)$ \\
\hline Age (mean \pm SD, y) & $28.7 \pm 7.52$ & $26.2 \pm 7.2$ \\
\hline Duration of epilepsy (mean \pm SD, y) & $12.0 \pm 9.1$ & $9.3 \pm 5.8$ \\
\hline Duration of AED therapy (mean \pm SD, y) & $5.5 \pm 4.8$ & $4.5 \pm 3.1$ \\
\hline \multicolumn{3}{|l|}{ Type of epilepsy } \\
\hline Generalized & $4(13.8 \%)$ & $2(7.7 \%)$ \\
\hline Partial & $25(86.2 \%)$ & $24(92.3 \%)$ \\
\hline \multicolumn{3}{|l|}{ Type of dugs } \\
\hline CBZ & 11 & 8 \\
\hline PHT & 11 & 4 \\
\hline OXCZ & 2 & 3 \\
\hline $\mathrm{CBZ}+\mathrm{CLB}$ & 3 & 7 \\
\hline $\mathrm{PHT}+\mathrm{CLB}$ & 2 & 1 \\
\hline $\mathrm{OXCZ}+\mathrm{CLB}$ & - & 3 \\
\hline
\end{tabular}

Abbreviations: AED, antiepileptic drug; CBZ, carbamazepine; CLB, clobazam; MWE, men with epilepsy; OXCZ, oxcarbazepine; PHT, phenytoin; SD, standard deviation; WWE, women with epilepsy. 
12 in luteal phase) are summarized in - Table 1. The mean age ( \pm standard deviation) of MWE and WWE was 28.7 years $( \pm 7.52)$ and 26.2 years $( \pm 7.2)$, respectively. The mean age of control population for men and women was $28.1( \pm 5.2)$ and 25.4 years (8.5), respectively.

Partial epilepsy was the most common type of epilepsy in our study (-Table 1). It constituted $86.2 \%$ and $92.3 \%$ in MWE and WWE, respectively. Mean duration of epilepsy was similar in both the groups (MWE and WWE: 12 and 9.8 years, respectively), and the mean duration of EIAED therapy was 5.5 and 4.5 years, respectively. In MWE 24 (83\%) were on monotherapy and 5 (17\%) were on polytherapy (- Table 1). In WWE 15 (57.7\%) were on monotherapy and $11(42.3 \%)$ were on polytherapy.

Males: Out of the 29 MWE, 14 (48.2\%) had a score of $\leq 21$ suggestive of sexual dysfunction. Fifteen MWE reported normal sexual function (a score of $\geq 22$ ). The detailed subanalysis was not performed in view of the limited number of patients. This was part of another study.

Unpaired Student's t-test results showed significant decrease in TT/LH $(p<0.0001)$, FT $(p<0.0001)$, BAT $(p<0.0001)$, DHEAS $(p=0.005)$, progesterone $(p<0.0001)$, significant increase in $\mathrm{E}_{2}(p<0.0001)$, and no significant difference seen in TT ( $p=0.247)$ in MWE as compared with controls (-Table 2).

Mann-Whitney $U$ test results showed significant increase in FSH $(p=0.022)$, LH $(p<0.0001)$, PRL $(p=0.022)$, SHBG $(p<0.0001)$, significant decrease in FAI $(p<0.0001)$, and no significant difference in LH/FSH $(p=0.26)$ in MWE as compared with controls ( $\mathbf{- T a b l e ~} 2$ ).

Females: Out of the $26 \mathrm{WWE}$, there were 9 (34\%) women with irregular menstrual cycle. The menstrual cycle was defined as irregular when the variation in the cycle is more than 8 days (Kippley et al, 1996). None of the control population suffered with menstrual irregularity.
An unpaired Student's t-test results showed significant increase in LH/FSH ( $p=0.0029)$, SHBG $(p<0.0001)$, significant decrease in FT $(p<0.0001)$, and no significant difference in $\mathrm{E}_{2}(p=0.3115)$ in WWE as compared with controls (-Table 3).

Mann-Whitney $U$ test results showed significant increase in FSH $(p=0.0262)$, LH $(p=0.0002)$, PRL $(p=0.0353)$, significant decrease in $\mathrm{E}_{2} / \mathrm{SHBG}(p<0.0001)$, progesterone $(p=0.0007)$, BAT $(p<0.0001)$, DHEAS $(p<0.0001)$ FAI $(p<0.0001)$, and no significant difference in TT $(p=0.0852)$ in WWE as compared with controls (- Table 3 ).

\section{Discussion}

Men and women with epilepsy appear to have an increased incidence of sexual dysfunction than patients with other chronic neurologic illnesses, with the dysfunction being manifested primarily as diminished sexual desire and potency. Sexual dysfunction affects 30 to $66 \%$ of MWE and 14 to $50 \%$ of WWE. ${ }^{4}$ MWE have sexual complaints that include lack of spontaneous morning penile tumescence, anorgasmia, and erectile difficulties whereas women report dyspareunia and vaginismus.

Antiepileptic drugs like such as carbamazepine (CBZ), phenytoin (PHT), and barbiturates induce cytochrome P450 enzyme system. This property of these EIAEDs has a substantial bearing upon the hormone profile and adversely affects sexual functions and fertility. However, it has not been studied in an Indian population.

In our study in MWE, FSH and LH levels were significantly higher than in controls. High FSH concentration that is more than 95th percentile of the controls was seen in 34\% patients, similar to the findings by Hamed et al 2007 reflecting an impairment of spermatogenesis. ${ }^{5}$

Table 2 Serum hormone levels in men with epilepsy and age-matched controls

\begin{tabular}{|c|c|c|c|c|c|}
\hline \multirow[t]{2}{*}{ Variable } & \multicolumn{4}{|c|}{ Controls $(n=29)$} & \multirow[t]{2}{*}{$p$-Value } \\
\hline & Mean \pm SD & 5-95th percentile & Mean \pm SD & 5-95th percentile & \\
\hline TT (ng/mL) & $4.5 \pm 1.9$ & $1.5-7.6$ & $4.9 \pm 1.4$ & $2.9-7.2$ & 0.247 \\
\hline TT/LH & $0.59 \pm 0.29$ & $0.19-1.15$ & $1.05 \pm 0.34$ & $0.57-1.58$ & $<0.0001$ \\
\hline FT (\%) & $1.19 \pm 0.36$ & $0.52-1.70$ & $2.33 \pm 0.5$ & $1.69-3.49$ & $<0.0001$ \\
\hline BAT (\%) & $31.7 \pm 10.2$ & $11.4-46.3$ & $57.8 \pm 9.6$ & $44.3-76.4$ & $<0.0001$ \\
\hline DHEAS $(\mu \mathrm{g} / \mathrm{dL})$ & $190 \pm 122$ & $49-414$ & $284 \pm 121$ & $113-499$ & 0.005 \\
\hline Estradiol (pg/mL) & $38.8 \pm 11.9$ & $24.7-60.1$ & $28.2 \pm 9.3$ & $11.1-45.5$ & $<0.0001$ \\
\hline \multirow[t]{2}{*}{ Progesterone (ng/mL) } & $0.263 \pm 0.193$ & $0.030-0.657$ & $0.519 \pm 0.184$ & $0.229-0.835$ & $<0.0001$ \\
\hline & Median & 25-75th percentile & Median & 25-75th percentile & \\
\hline $\mathrm{FSH}(\mathrm{mlU} / \mathrm{mL})$ & 4.3 & $3.4-8.6$ & 3.4 & $2.8-4.5$ & 0.022 \\
\hline $\mathrm{LH}(\mathrm{mlU} / \mathrm{mL})$ & 7.9 & $5.9-10.0$ & 5 & $3.9-5.9$ & $<0.0001$ \\
\hline Prolactin $(\mu \mathrm{lU} / \mathrm{mL})$ & 196 & $147-295$ & 158 & $126-226$ & 0.022 \\
\hline SHBG (nmol/L) & 68 & $50-79$ & 25 & $20-34$ & $<0.0001$ \\
\hline FAI & 22.2 & $10.2-33.9$ & 62.6 & $49.2-84.1$ & $<0.0001$ \\
\hline
\end{tabular}

Abbreviations: BAT, bioactive testosterone; DHEAS, dehydroepiandrosterone sulfate; FAI, free androgen index; FSH, follicular-stimulating hormone; FT, free testosterone; LH, luteinizing hormone; MWE, men with epilepsy; SD, standard deviation; SHBG, sex hormone-binding globulin; $\mathrm{TT}$, total testosterone. 
Table 3 Serum hormone levels in women with epilepsy and age-matched controls

\begin{tabular}{|c|c|c|c|c|c|}
\hline \multirow[t]{2}{*}{ Variables } & \multicolumn{2}{|c|}{ Patients $(n=26)$} & \multicolumn{2}{|c|}{ Controls $(n=26)$} & \multirow[t]{2}{*}{$p$-Value } \\
\hline & Mean \pm SD & 5-95th percentile & Mean \pm SD & 5-95th percentile & \\
\hline $\mathrm{LH} / \mathrm{FSH}$ & $1.71 \pm 0.93$ & $0.38-3.68$ & $1.07 \pm 0.48$ & $0.42-2.11$ & 0.0029 \\
\hline $\mathrm{E}_{2}(\mathrm{pg} / \mathrm{mL})$ & $149 \pm 105.6$ & $14.92-402.7$ & $125.2 \pm 54$ & $52.72-225.6$ & 0.3115 \\
\hline SHBG (nmol/L) & $184 \pm 69.4$ & $74.7-311.3$ & $51.6 \pm 23.5$ & $17.6-92.0$ & $<0.0001$ \\
\hline \multirow[t]{2}{*}{ FT (\%) } & $0.55 \pm 0.22$ & $0.30-1.03$ & $1.49 \pm 0.49$ & $0.87-2.53$ & $<0.0001$ \\
\hline & Median & 25-75th percentile & Median & 25-75th percentile & \\
\hline $\mathrm{FSH}(\mathrm{mlU} / \mathrm{mL})$ & 5.8 & $4.6-9.9$ & 4.6 & $2.8-7.1$ & 0.0262 \\
\hline $\mathrm{LH}(\mathrm{mlU} / \mathrm{mL})$ & 8.5 & $6.1-15.0$ & 3.85 & $2.6-8.6$ & 0.0002 \\
\hline Prolactin $(\mu \mathrm{lU} / \mathrm{mL})$ & 314.3 & $213.3-452.5$ & 239.1 & $172.9-286.2$ & 0.0353 \\
\hline $\mathrm{E}_{2} / \mathrm{SHBG}$ & 0.0028 & $0.0013-0.0047$ & 0.00763 & $0.0059-0.0149$ & $<0.0001$ \\
\hline Progesterone (ng/mL) & 0.275 & $0.06-2.42$ & 4.175 & $0.71-12.44$ & 0.0007 \\
\hline TT (ng/mL) & 0.15 & $0.03-0.25$ & 0.20 & $0.13-0.29$ & 0.0852 \\
\hline BAT (\%) & 10.75 & $9.3-16.28$ & 33.85 & $25.03-45.95$ & $<0.0001$ \\
\hline FAI & 0.0026 & $0.0006-0.0048$ & 0.0115 & $0.008-0.032$ & $<0.0001$ \\
\hline DHEAS $(\mu \mathrm{g} / \mathrm{dL})$ & 78.9 & $37.7-126.5$ & 186.9 & $121.1-252.2$ & $<0.0001$ \\
\hline
\end{tabular}

Abbreviations: BAT, bioactive testosterone; DHEAS, dehydroepiandrosterone sulfate; $\mathrm{E}_{2}$, estradiol; FAl, free androgen index; FSH, follicular-stimulating hormone; FT, free testosterone; LH, luteinizing hormone; SD, standard deviation; SHBG, sex hormone-binding globulin; TT, total testosterone; WWE, women with epilepsy.

Total testosterone concentrations were not altered significantly in our study except in three MWE in whom TT levels were below the reference range indicating a clinically relevant testicular disturbance. FT and BAT were significantly decreased in MWE $(p<0.0001)$ than in controls. In 27 (93\%) patients, FT levels were below the 5th percentile of controls. The documented increase in SHBG is the most plausible explanation for this finding. The concomitant finding of decreased FT and an increased SHBG level has previously been reported. Lowered FT levels might have an impact on the sexual drive leading to testicular failure and hypogonadism. Fenwick et al demonstrated a relationship between decreased potency and low FT levels. ${ }^{6}$ Testosterone supplementation can be an effective treatment for hypogonadal MWE and sexual dysfunction. It may improve not only sexual function but also mood and even seizure frequency.

There was significant decrease in FAI in MWE compared with controls $(p<0.0001)$. FAI is an index that indirectly estimates the level of testosterone binding to albumin, ${ }^{9}$ which is potentially active in target tissues. Decreased FAI indicates a potentially lower portion of BAT and is associated with diminished sexual function in men.

TT/LH, a sensitive marker for Leydig's cell function, was significantly lower in $52 \%$ of MWE than in controls in our study. Despite an increased release of LH, Leydig's cells are not able to further increase testosterone synthesis, causing a decreased $\mathrm{TT} / \mathrm{LH}$. This inhibition of testosterone synthesis is probably due to EIAEDs, which has been demonstrated in other studies. ${ }^{10}$

In our study, SHBG levels were significantly higher in MWE than controls, and in 26 (90\%) MWE, these levels exceeded 95th percentile of controls $(p<0.0001)$. EIAEDs induced the hepatic metabolism of sex hormones and also accelerated the synthesis of SHBG. ${ }^{3}$
$\mathrm{E}_{2}$ levels were significantly higher in MWE $(p<0.0001)$ than controls similar to study by Herzog et al, which found serum $E_{2}$ levels to be significantly higher in PHT-treated MWE than among untreated or normal controls. ${ }^{11}$ Murialdo et al stated that though increased serum $\mathrm{E}_{2}$ levels constitute only $1 \%$ of total reproductive steroids, it exerts one-half of the negative feedback on the hypothalamo-pituitary-axis. ${ }^{12}$ Such increase could have a disproportionately large negative feedback effect on gonadotropin production. Also, by producing premature aging of the hypothalamic arcuate nucleus and by lowering BAT, it contributes to hypogonadism.

The serum concentration of DHEAS was significantly lower in MWE $(p=0.005)$ compared with controls, which is in concordance with previous studies. Several mechanisms may lead to this effect. One reason for the reduction in DHEAS, described by Levesque et al, is that of a direct toxic effect of PHT and CBZ on the adrenal glands..$^{13}$ This finding supports the potential risk of erectile dysfunction in MWE treated with EIAEDs, as DHEAS triggers the production of endothelial nitric oxide, ${ }^{14}$ a signaling molecule that has a role in mediating erectile function.

According to Mölleken et al, sexual dysfunction in MWE is directly related to the altered sex hormone levels (raised levels of FSH or SHBG and decreased levels of DHEAS and BAT). ${ }^{15}$ This is almost similar to findings in our study that shows increased FSH, SHBG levels and decreased DHEA and BAT levels. BAT is thought to contribute to the level of sexual interest. Moreover, decreased level of BAT is assumed to have a negative impact on sperm quality, which may affect spermatogenesis. In another in vitro study, EIAEDs were associated with decreased sperm motility indicating a direct effect of these drugs on sperm quality, ${ }^{16}$ which may contribute to decreased fertility. Since BAT decreases with age more 
rapidly in MWE on EIAEDs, ${ }^{17}$ older men may be at an even higher risk for sexual dysfunction.

In WWE, we found statistically significant increase in PRL levels when compared with controls. Three of them had markedly elevated PRL levels that was probably stress induced. The fact that these three patients reported regular menstrual cycles supplements this assumption. In two of three, ovulatory cycle was documented and proved by normal progesterone levels in the luteal phase. Therefore, we further deduced that anovulation in WWE do not seem to be caused by hyperprolactinemia. No consistent abnormalities in basal PRL levels have been reported in women taking PHT or CBZ for epilepsy. ${ }^{18}$

SHBG levels were significantly higher than in the controls ( $p<0.0001$ ). Identical findings have previously been reported. $E_{2}$ did not show any significant difference between groups, but there was significant decrease in $\mathrm{E}_{2} / \mathrm{SHBG}$ $(p<0.0001)$ that is indirectly an estimate of bioactive $\mathrm{E}_{2}$. Decreased levels of $E_{2} / S H B G$ had previously been reported in WWE on EIAEDs. ${ }^{18}$ Increase in SHBG and resulting reduction in serum-free $E_{2}$ may interfere with the feedback regulation of pituitary secretion, which may lead to anovulation and menstrual disorders.

We observed significant decrease in progesterone levels in WWE compared with controls ( $p=0.0007)$. Decreased progesterone concentrations were observed in 4 of 12 patients in luteal phase. Relatively low levels of progesterone in luteal phase also point toward possibility of anovulatory cycles and/or poor functioning of corpus luteum. EIAEDs have also been reported to increase the level of SHBG that decreases free progesterone levels. ${ }^{19}$ In our study, women had inadequate luteal function and abnormal LH levels suggesting pituitary ovarian dysregulation.

There was significant decrease in FT in WWE compared with controls ( $p<0.0001)$. It is well established that EIAEDs by inducing hepatic cytochrome P450 3A4 can lower FT. There was significant decrease in BAT in WWE compared with controls $(p<0.0001)$. BAT, known to be an important hormone for male sexual functioning, also correlates with degree of sexual interest in women. There was significant decrease in FAI in WWE compared with controls $(p<0.0001)$. In fact, decrease in FAI may protect against polycystic ovarian syndrome (PCOS) in susceptible women, and conversely, increase in FAI may worsen a PCOS. ${ }^{20}$ While treating PCOS-susceptible WWE, due consideration should be given to these factors and EIAEDs may counter such adverse effects of valproate.

In WWE there was significant decrease in DHEAS $(p<0.0001)$, which has been previously reported though its clinical significance is unknown. ${ }^{21}$

Morrell et al stated that WWE had lower sexual satisfaction when compared with women treated with lamotrigine and gabapentin. ${ }^{22}$ A recent study done in India by Sukumaran et al concluded that infertility was most common in women who were on EIAEDs compared with those without EIAEDs (7.1\%). ${ }^{23}$ They found that risk of infertility was $31.8 \%$ with one AED, $40.7 \%$ with two AEDs, and $60.3 \%$ with three or more AEDs. ${ }^{22}$
Reported changes in sex hormones secondary to EIAEDs exposure may be reversible. This is supported by data from a small prospective study conducted by Lossius et al in which seizure-free MWE and WWE were tapered off EIAEDs and after 4 months demonstrated significant decrease in SHBG levels and increase in TT, FAI, FT, and $\mathrm{E}_{2} / \mathrm{SHBG}$, suggesting that potential changes in sex steroid levels are reversible. ${ }^{20}$ Owing to cross-sectional design of our study, we cannot comment about the reversibility of these changes.

The reason why only few individuals are susceptible to the hormonal alterations is not clear. Genetic variability in drug response and variable response of CYP isoenzymes to particular inducer could be responsible for some of the conflicting data available in the literature. Polymorphisms in CYP450 genes involved with sex hormone metabolism directly determine levels of circulating hormones. Future studies should evaluate the independent and joint effects of CYP450 genetic polymorphisms at each step of the sex hormone metabolic pathway in patients on EIAEDs.

\section{Conclusions}

Our study clearly demonstrates that EIAEDs have an adverse effect on reproductive health in both MWE and WWE. The hormone abnormalities described may provide insight into mechanisms and potential treatments for specific types of sexual dysfunction. In prescribing EIAEDs to men and women of reproductive age group, a better understanding of its endocrine effects should be taken into consideration. Some of these changes may be reversible on withdrawal of EIAEDS, even after years of treatment. Awareness of these key factors would enable clinicians to give appropriate treatment guidelines that can reduce adverse health effects.

\section{Limitations and Future Considerations}

We could not analyze the effects of individual EIAEDs as some were on polytherapy. Semen analysis could have been done to strongly confirm our results. The endocrine effects of the new AEDs have not been assessed. Because of promising results of our study and disparate finding from other literature, further studies are warranted in epileptic patients on EIAEDs to evaluate the effects of CYP450 polymorphisms in genes at various steps of the sex hormone metabolic pathway.

\section{Highlights}

- Elevation of SHBG and $\mathrm{E}_{2}$ and decrease in TT/LH, FT, BAT, DHEAS, and progesterone were found in MWE.

- Elevation of SHBG and LH/FSH and decrease in progesterone, DHEAS, and $\mathrm{E}_{2} / \mathrm{SHBG}$ were observed in WWE.

- Enzyme inducing antiepileptic drugs induce CYP450 and other enzymes to cause these hormonal changes.

- This hormonal change explains the decreased libido, erectile dysfunction, and infertility seen in this group. 


\section{Authors' Contributions}

Dr. Shaik Jabeen and Dr. Shivalaxmi were mainly involved in the design of study, conduct of study, and also write-up. Dr. Prisicilla Chandran and Dr. Noor Jahan helped in data analysis and statistics. Dr. Ajith Cherian helped in only revising the manuscript. The IEC approval was only taken from Nizam's Institute of Medical Sciences.

\section{Conflict of Interest}

None.

\section{Acknowledgments}

We thank all the participants in this study for their cooperation and patience while carrying out the study, without whom this study could not have been possible nor could we be wiser without them.

\section{References}

1 Harden CL. Sexual dysfunction in women with epilepsy. Seizure 2008;17(2):131-135

2 Toone BK, Edeh J, Nanjee MN, Wheeler M. Hyposexuality and epilepsy: a community survey of hormonal and behavioural changes in male epileptics. Psychol Med 1989;19(4):937-943

3 Isojärvi JI, Pakarinen AJ, Ylipalosaari PJ, Myllylä VV. Serum hormones in male epileptic patients receiving anticonvulsant medication. Arch Neurol 1990;47(6):670-676

4 Jensen P, Jensen SB, Sørensen PS, et al. Sexual dysfunction in male and female patients with epilepsy: a study of 86 outpatients. Arch Sex Behav 1990;19(1):1-14

5 Hamed SA, Hamed EA, Shokry M, Omar H, Abdellah MM. The reproductive conditions and lipid profile in females with epilepsy. Acta Neurol Scand 2007;115(1):12-22

6 Fenwick PBC, Mercer S, Grant R, et al. Nocturnal penile tumescence and serum testosterone levels. Arch Sex Behav 1986;15(1):13-21

7 Herzog AG. Hormonal therapies: progesterone. Neurotherapeutics 2009;6(2):383-391

8 Chandran PA, Laxm MS, Shaik AJ, Mohammed N. Serum sex hormone levels-biomarkers of sexual dysfunction in men with epilepsy on enzyme inducing antiepileptic drugs. IJMB 2012;18(2):xx

9 Manni A, Pardridge WM, Cefalu W, et al. Bioavailability of albumin-bound testosterone. J Clin Endocrinol Metab 1985;61(4):705-710
10 Saunders M, Rawson M. Sexuality in male epileptics. J Neurol Sci 1970;10(6):577-583

11 Herzog AG, Seibel MM, Schomer DL, Vaitukaitis JL, Geschwind N. Reproductive endocrine disorders in men with partial seizures of temporal lobe origin. Arch Neurol 1986;43(4):347-350

12 Murialdo G, Galimberti CA, Fonzi S, et al. Sex hormones and pituitary function in male epileptic patients with altered or normal sexuality. Epilepsia 1995;36(4):360-365

13 Levesque LA, Herzog AG, Seibel MM. The effect of phenytoin and carbamazepine on serum dehydroepiandrosterone sulfate in men and women who have partial seizures with temporal lobe involvement.J Clin Endocrinol Metab 1986;63(1):243-245

14 Liu D, Dillon JS. Dehydroepiandrosterone activates endothelial cell nitric-oxide synthase by a specific plasma membrane receptor coupled to Galpha (i2,3) J Biol Chem 2002;277 (24):21379-21388

15 Mölleken D, Richter-Appelt H, Stodieck S, Bengner T. Sexual quality of life in epilepsy: correlations with sex hormone blood levels. Epilepsy Behav 2009;14(1):226-231

16 Chen SS, Shen MR, Chen TJ, Lai SL. Effects of antiepileptic drugs on sperm motility of normal controls and epileptic patients with long-term therapy. Epilepsia 1992;33(1):149-153

17 Victor A, Lundberg PO,Johansson ED. Induction of sex hormone binding globulin by phenytoin. BMJ 1977;2(6092):934-935

18 Isojärvi JI, Repo M, Pakarinen AJ, Lukkarinen O, Myllylä VV. Carbamazepine, phenytoin, sex hormones, and sexual function in men with epilepsy. Epilepsia 1995;36(4):366-370

19 Rättyä J, Turkka J, Pakarinen AJ, et al. Reproductive effects of valproate, carbamazepine, and oxcarbazepine in men with epilepsy. Neurology 2001;56(1):31-36

20 Lossius MI, Taubøll E, Mowinckel P, Mørkrid L, Gjerstad L. Reversible effects of antiepileptic drugs on reproductive endocrine function in men and women with epilepsy-a prospective randomized double-blind withdrawal study. Epilepsia 2007;48(10):1875-1882

21 Macphee GJ, Larkin JG, Butler E, Beastall GH, Brodie MJ. Circulating hormones and pituitary responsiveness in young epileptic men receiving long-term antiepileptic medication. Epilepsia 1988;29(4):468-475

22 Morrell MJ, Flynn KL, Doñe S, Flaster E, Kalayjian L, Pack AM. Sexual dysfunction, sex steroid hormone abnormalities, and depression in women with epilepsy treated with antiepileptic drugs. Epilepsy Behav 2005;6(3):360-365

23 Sukumaran SC, Sarma PS, Thomas SV. Polytherapy increases the risk of infertility in women with epilepsy. Neurology 2010;75(15):1351-1355 